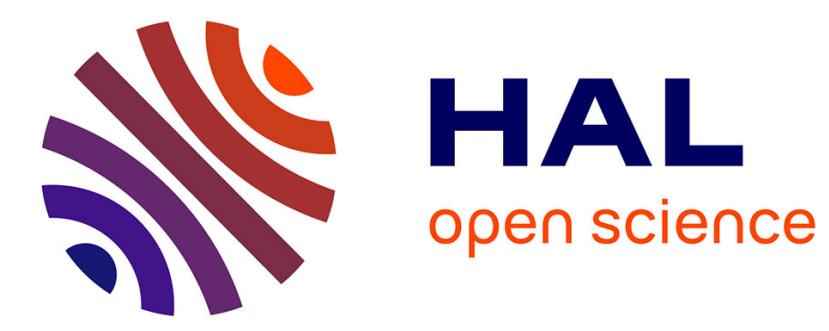

\title{
Espace et religion: chrétiens et musulmans en Albanie du Sud
}

Gilles de Rapper

\section{To cite this version:}

Gilles de Rapper. Espace et religion: chrétiens et musulmans en Albanie du Sud. Études balkaniques. Cahiers Pierre Belon, 2002, 9, pp.17-39. halshs-00258572

\section{HAL Id: halshs-00258572 \\ https://shs.hal.science/halshs-00258572}

Submitted on 27 Jun 2008

HAL is a multi-disciplinary open access archive for the deposit and dissemination of scientific research documents, whether they are published or not. The documents may come from teaching and research institutions in France or abroad, or from public or private research centers.
L'archive ouverte pluridisciplinaire HAL, est destinée au dépôt et à la diffusion de documents scientifiques de niveau recherche, publiés ou non, émanant des établissements d'enseignement et de recherche français ou étrangers, des laboratoires publics ou privés. 
Article publié dans Études balkaniques. Cahiers Pierre Belon 9 (2002), p. 17-39.

\author{
ESPACE ET RELIGION : CHRÉTIENS ET MUSULMANS EN ALBANIE DU SUD
}

Gilles de Rapper

[19] En dehors de celles à caractère historique, les études sur la religion en Albanie se divisent en deux groupes, selon qu'elles traitent de l'articulation entre appartenance religieuse et identité nationale ou de la religion populaire. On peut y voir la conséquence du paysage religieux albanais et de ses développements récents : largement islamisés pendant la période ottomane, les Albanais accèdent à l'indépendance en 1912 dans le cadre d'un État albanais qui regroupe environ $70 \%$ de musulmans (principalement sunnites et bektachis) et $30 \%$ de chrétiens (orthodoxes et catholiques), et dans un contexte balkanique où le christianisme, en particulier orthodoxe, se présente comme le défenseur des nations balkaniques et le garant de leur intégrité malgré les siècles de "joug turc". Dans le cas albanais, où les promoteurs de l'idée nationale se rencontrent tant parmi les musulmans que parmi les chrétiens, la question de l'articulation entre identité nationale et identité religieuse est aussi celle de la place de l'islam, et donc de l'héritage ottoman, dans la société albanaise.

On ne cherchera pas ici à répondre directement à cette question ; on ne posera pas non plus la question de l'articulation entre l'islam et le christianisme d'une part, et la religion populaire, d'autre part. La position de la recherche albanaise pendant la période communiste et pour une part aujourd'hui encore, est en effet que la véritable religion des Albanais, celle qui peut prétendre au caractère de religion nationale, ne doit pas être recherchée dans les grandes religions importées que sont l'islam et le christianisme, mais dans la religion populaire, telle qu'elle est observée par les [20] ethnologues, du moins avant qu'elle ne soit combattue par le régime communiste au titre de superstition dépassée.

Je chercherai au contraire, par l'exemple d'une région du sud-est albanais, à montrer comment discours et représentations de la religion et des communautés religieuses sont le lieu 
d'une définition de l'autre assez peu concernée par l'identité nationale. L'argument est que les relations entre les deux communautés religieuses en présence (musulmane et chrétienne orthodoxe) s'articulent à l'organisation spatiale et sociale de la région, et que la différence religieuse structure la société locale au même titre que d'autres oppositions, en particulier que celle entre Nord et Sud.

Le matériel sur lequel repose cet article a été recueilli en 1995 et 1996 principalement, dans le Devoll, un district du sud-est albanais regroupant une quarantaine de villages autour de la petite ville de Bilisht. Les habitants y sont majoritairement musulmans, seuls trois ou quatre villages étant entièrement chrétiens (dont l'un, Vërnik, relève de la minorité macédonienne d'Albanie). Les lieux de culte en activité y sont rares et la pratique limitée par l'absence de personnel religieux. Chacun sait pourtant que, quelles que soient ses convictions personnelles, il appartient à une communauté religieuse plutôt qu'à une autre, et que les relations qu'il entretient avec les autres sont déterminées en grande partie par leur appartenance à l'une ou l'autre des communautés.

\section{Communautés religieuses}

Il est dans un premier temps nécessaire de préciser ce qu'il faut entendre par " communautés religieuses" et distinguer pour cela le niveau officiel, celui de l'État, du niveau local, celui de l'organisation sociale des villages du district en question. Depuis sa création en 1913 jusqu'à aujourd'hui, l'État albanais est un État laïque, qui ne reconnaît aucune religion officielle. La tendance actuelle est au maintien de la séparation entre les affaires de l'État et celles de la religion, et le retour au pluralisme politique au début des années quatre-vingt-dix s'est par exemple accompagné d'une restriction selon laquelle les partis politiques à base ethnique ou religieuse sont interdits. Les croyants sont donc organisés au sein d'organisations religieuses qui, tout en ayant un caractère national, sont indépendantes de l'État albanais. Il s'agit, dans le cadre de la population qui nous intéresse ici, de la "Communauté musulmane albanaise", appelée en albanais Komuniteti mysliman shqiptar, et de "l'Église orthodoxe autocéphale albanaise", en albanais Kisha ortodokse autoqefale shqiptare (reconnue par Constantinople en 1937). Ces deux communautés sont censées gérer et entretenir les lieux de [21] culte et le personnel religieux ${ }^{1}$. Depuis 1999, les relations entre les communautés institutionnelles et l'État sont réglées par le Comité étatique des cultes (Komiteti shtetëror $i$ kulteve), qui dépend du Conseil des ministres.

\footnotetext{
${ }^{1}$ L'organisation institutionnelle des musulmans a fait l'objet de plus d'attention que celle des chrétiens. Pour les premiers, voir POPOVIC Alexandre, 1986. L'Islam balkanique. Wiesbaden, Otto Harrassowitz et LAKSHMANLEPAIN Rajwantee, 2001. Albanie : les enjeux de la réislamisation. in BOUGAREL Xavier et CLAYER Nathalie (dir.), 2001. Le nouvel islam balkanique. Les musulmans acteurs du post-communisme, 1990-2000. Paris, Maisonneuve \& Larose, 133-176. Pour les seconds, voir QIRIAZI Dhori, 2000. Krishterimi në Shqipëri [Le christianisme en Albanie]. Tiranë, Argeta LMG.
} 
Après la complète disparition des communautés officielles pendant le communisme, leur influence dans les campagnes et les régions les plus périphériques de l'espace albanais n'est pas aujourd'hui entièrement rétablie. Dans le district du Devoll, en 1996, une seule mosquée fonctionnait régulièrement (celle de la ville de Bilisht, construite en 1995) et la plupart des églises chrétiennes, si elles étaient ouvertes aux fidèles, devaient se passer de prêtre, tant la pénurie de personnel religieux était grande. Dans les villages musulmans, les mosquées n'ont en général pas été reconstruites (à l'exception de quelques tekkes bektachies et halvéties), et le seul spécialiste religieux du village est le hodja (en albanais hoxhè), qui n'est souvent pas reconnu par la Communauté musulmane albanaise, et dont la connaissance de l'islam est très approximative. Au total, la présence des communautés religieuses officielles sur le terrain est très faible, et elle entre souvent en concurrence avec les missions étrangères, catholiques ou protestantes, dont les activités dans les zones reculées sont en général plus suivies.

Les deux communautés religieuses dont il est question ici ne sont donc pas les communautés institutionnelles; il s'agit plutôt de communautés informelles, qui reposent sur le simple fait que les gens qui en font partie partagent consciemment une même appartenance religieuse. En d'autres termes, si les communautés officielles constituent plutôt, dans le langage wébérien, des associations, c'est-à-dire des groupes formés en vue d'accomplir certains objectifs, les communautés informelles reposent sur un phénomène de communalisation, c'està-dire sur le sentiment d'appartenance commune qu'engendre le partage de certaines caractéristiques. Ensuite, ces communautés informelles, au nombre de deux dans le Devoll, ne fonctionnent qu'au niveau local, [22] c'est-à-dire au niveau du district ou de la région : cela signifie que les musulmans du Devoll ne se sentent pas solidaires de tous les musulmans d'Albanie, pour ne pas parler des musulmans du reste du monde; dès que l'on quitte le niveau local au contraire, musulmans et chrétiens du Devoll font bloc pour s'opposer tous ensemble, sur la base d'une identité régionale, au reste de l'Albanie. Les musulmans du Devoll, par exemple, accusent ainsi les autres musulmans albanais d'être "fanatiques", c'est-à-dire d'accorder trop d'importance à la religion, alors qu'eux-mêmes ne font aucune différence entre chrétiens et musulmans et, selon eux, s'accommodent très bien de leur coexistence avec les chrétiens. Dans le cadre du district cependant, comme on le verra par la suite, la coexistence entre chrétiens et musulmans n'est pas exempte de heurts et de ressentiments. L'importance du critère religieux dans la construction des identités collectives doit donc être rapportée au niveau d'analyse que l'on privilégie. Dans ce sens, on peut reconnaitre que l'appartenance religieuse joue un rôle secondaire dans la construction de l'identité nationale en Albanie, tout en reconnaissant par ailleurs son importance dans la construction des identités collectives en situation locale.

Il faut d'autre part tenir compte du décalage qui existe entre l'organisation sociale, telle qu'elle peut être observée de l'extérieur, et les discours que les agents eux-mêmes portent sur la société ; dans le cas des communautés religieuses locales, ce décalage peut-être important, dans la mesure où les communautés ne sont pas reconnues de manière institutionnelle : leur contenu 
et leurs limites peuvent donc varier selon le point du vue que l'on retient. Ainsi, à écouter les discours tenus par la plupart des chrétiens et des musulmans, on peut avoir l'impression d'une rupture totale entre les deux communautés locales, alors que l'observation révèle au contraire une très large communauté de modes d'existence, de langue et de références culturelles. Par exemple, la langue parlée par les chrétiens du Devoll ne diffère pas de celles des musulmans : on y rencontre les mêmes emprunts ottomans et italiens, et les emprunts grecs, malgré la proximité de la frontière grecque, sont à peine plus nombreux chez les chrétiens. D'autre part, alors qu'au niveau institutionnel et religieux les musulmans se répartissent en sunnites, bektachis et autres ordres mystiques, les représentations de la religion en relation avec l'organisation sociale locale ne considèrent qu'une seule grande catégorie de "musulmans", opposée à celle des chrétiens.

L'examen de la terminologie révèle également le décalage entre les communautés informelles et les communautés [23] institutionnelles : les premières ne sont jamais désignées par le mot komunitet, mais tant chez les musulmans que chez les chrétiens orthodoxes, par l'emprunt italien $f e$, parfois en concurrence avec les mots albanais besë et besim qui désignent cependant plus la foi et la croyance que la religion. Le mot fe désigne à la fois la religion comme phénomène général et comme religion particulière, liée à une communauté déterminée. On parle donc de feja e krishterë, "la religion chrétienne", et de feja myslymane ou, plus fréquemment encore, de feja turke, "la religion musulmane, ou turque". Dans ces expressions, le mot fe désigne à la fois un ensemble de pratiques et de croyances, et la communauté qui s'identifie à ces pratiques et croyances.

\section{La dénomination}

Lorsqu'il s'agit de parler de la communauté en elle-même et de la nature des gens qui la composent, le mot fe est souvent remplacé par d'autres termes évoquant une origine et une appartenance communes. Il s'agit des mots racë, emprunté à l'italien razza (peut-être par l’intermédiaire du grec), et komb, néologisme formé sur l’arabe kawm, qui désigne dans la langue standard la "nation". On peut ainsi entendre les expressions de "race chrétienne" (racë $e$ krishterè) ou "race des chrétiens" (raca e krishterëve), ou encore celle de "nation turque" (komb turk), cette dernière ne faisant référence qu'aux musulmans albanais. Cette particularité terminologique est liée au fait que, bien souvent, les discours portés par l'une ou l'autre communauté visent à faire effectivement des deux communautés des groupes relativement fermés, définis par des origines différentes et par des caractéristiques que l'on cherche à faire passer pour " naturelles ", c'est-à-dire propres aux individus appartenant à l'une ou l'autre des communautés, mais sans avoir toujours de rapport avec le dogme auquel ils adhèrent. En ce sens, l'appartenance religieuse est présentée en termes de race et de consanguinité et comme inscrite dans la nature même des choses, et donc immuable. 
D'autre part, si les communautés religieuses sont au nombre de deux, musulmane et chrétienne, les ethnonymes qui les désignent sont plus nombreux. Il faut en effet souvent distinguer les ethnonymes utilisés par une communauté pour se désigner et ceux utilisés par l'autre communauté pour la désigner. Le terme généralement utilisé par les musulmans pour se désigner est celui [24] de myslyman ou mysliman'. C'est un terme officiel, retenu par la langue standard (dictionnaires, presse), et neutre. Tout musulman devrait se dire myslyman et être désigné comme myslyman par les autres. Les choses ne sont cependant pas aussi claires. D'abord, les musulmans, qu'ils soient croyants ou non, jeunes ou vieux, se disent souvent euxmêmes turcs (turk). Le terme est en général évité en face d'un étranger, à cause de la confusion possible avec l'ethnonyme national turc, exactement identique (turk), et les musulmans albanais insistent souvent sur le fait que, bien qu'ils soient musulmans, ils ne sont pas turcs, mais albanais. Mais quand la familiarité avec l'étranger fait tomber ces précautions, c'est bien le terme turk qui est utilisé le plus souvent, sans ambiguité avec l'ethnonyme national turc. D'ailleurs, comme la plupart des ethnonymes nationaux en albanais, ce dernier est généralement utilisé au singulier, alors que dans le contexte religieux le mot est mis au pluriel. On dit ainsi "le Turc" (Turku) ou "le Grec" (Greku), là où, en français, un pluriel serait utilisé : na pushtovi Turku ("le Turc nous a envahi"), vete de Greku ("aller chez le Grec", aller en Grèce). Dans le contexte religieux, on utilise au contraire le pluriel, comme dans la formule ne turqtë (ou ne turqit), " nous les turcs, les musulmans".

Le mot turk est également utilisé par les chrétiens pour désigner les musulmans. S'il est le plus souvent utilisé au pluriel, il prend en revanche une connotation nettement négative. Un musulman qui se dit lui-même turk, au milieu d'autres musulmans, ne supportera pas d'être appelé de la même manière par un chrétien, mais exigera l'emploi du terme neutre myslyman. Dans la bouche d'un chrétien en effet le mot sonne comme une insulte, dont la forme complète est turk i mutit, " musulman de merde ", particulièrement violente. De plus, l'emploi que font les chrétiens du mot turk joue beaucoup plus de l'ambiguïté avec l'ethnonyme national : quand un chrétien désigne les musulmans comme " turcs", il cherche à montrer que les musulmans ont une origine extérieure à l'Albanie et qu'ils ne sont pas aussi Albanais que les chrétiens, authentiques autochtones. Les habitants du village de Vërnik, près de Bilisht, qui sont orthodoxes et bilingues (ils parlent albanais et macédonien et se disent eux-mêmes Macédoniens, maqedonas), utilisent, en albanais, le mot turk pour désigner les Albanais. Les chrétiens utilisent un autre terme pour désigner les [25] musulmans, qui leur paraît plus neutre et respectueux, même s'il semble inconnu des musulmans, c'est muamedan ${ }^{3}$.

Pour se désigner eux-mêmes, les chrétiens utilisent deux termes. L'un, le plus fréquent, est $i$ krishterë, l'autre est ortodoks. Le premier, avec son article préposé, est sans doute le plus ancien. Le sens du mot $i$ krishterë est clair en albanais, il fait référence au Christ (Krisht). Il désigne en principe tous les chrétiens, mais se limite de fait dans le Devoll aux chrétiens

\footnotetext{
${ }^{2}$ Le dictionnaire a mysliman, mais c'est myslyman qui est entendu dans le Devoll.

${ }^{3}$ Le dictionnaire a muhamedan, mais le son $b$ est absent de la plupart des mots dans le Devoll.
} 
orthodoxes, à l'exclusion des catholiques, très peu nombreux et d'arrivée récente dans la région. Ces derniers sont appelés katolik, et l'ensemble des chrétiens (orthodoxes et catholiques) est parfois désigné par le terme kristian, utilisé tant par les chrétiens que par les musulmans, et qui passe pour un mot plus recherché.

Le mot ortodoks n'est employé que par les chrétiens, les musulmans n'utilisent en général que $i$ krishterë. Lorsqu'un musulman utilise cependant le mot ortodoks, c'est pour montrer de la distance ou du respect, pour marquer de façon insistante qu'il ne cherche pas à placer la différence entre musulmans et chrétiens ailleurs que sur l'appartenance religieuse. La dénomination officielle de l'église orthodoxe albanaise est d'ailleurs Kisha ortodokse autoqefale shqiptare ("Église orthodoxe autocéphale albanaise"). Ortodoks fait ainsi plus référence à l'organisation elle-même, à la communauté organisée, qu'à ses membres et à leur mode de vie, qui sont le plus souvent qualifiés de $i$ krishterë.

Le mot le plus souvent utilisé par les musulmans pour désigner les chrétiens, y compris en leur présence, est celui de kaur. Du turc gawnr, lui-même dérivé de l'arabe kâfir, il signifie " infidèle, incroyant" et était utilisé dans l'empire ottoman par les musulmans pour désigner les chrétiens ${ }^{4}$. Il est aujourd'hui considéré comme offensant par les chrétiens albanais (la dénomination complète est souvent kaur $i$ derrit, "chrétien du porc"), mais pour beaucoup de musulmans il est la seule façon de désigner les chrétiens, sans pour autant chercher à les insulter. Le sens d"“ infidèle" (i pafe) n'est pas clairement reconnu en albanais, du moins par les musulmans qui l'emploient ${ }^{5}$, et kaur désigne seulement les chrétiens orthodoxes, [26] non pas comme non musulmans, mais spécifiquement comme orthodoxes. Le mot n'est d'ailleurs pas utilisé pour les catholiques, albanais ou autres, mais désigne également les chrétiens orthodoxes non albanais, notamment ceux de Grèce. Le fait que Macédoniens et Grecs sont également appelés kaur atténue la dimension religieuse de l'ethnonyme et, pour beaucoup de musulmans, être kaur ne signifie pas seulement ne pas être musulman, ou appartenir à une autre communauté religieuse. C'est aussi, en tant que membre d'une autre communauté, être fondamentalement différent des musulmans, par le mode de vie certes, mais aussi par "nature", en tant que "race" (raci), et parfois également par la langue. C'est ainsi que les Macédoniens peuvent être dits parler une langue autre que l'albanais, non pas en tant que membres d'une autre communauté linguistique, mais en tant que kaur.

Les ethnonymes utilisés pour désigner les différentes communautés religieuses ne sont donc pas exclusivement religieux. Ils prennent un sens plus large et élargissent la communauté religieuse pour en faire une communauté de langue ou, plus généralement, de " race ".

\footnotetext{
${ }^{4}$ W. BJÖRKMAN, in Encyclopédie de l'islam² IV : 425-427.

${ }^{5}$ Les chrétiens sont en général nettement plus conscients de l'origine et de la signification du mot kaur, qu'ils emploient rarement pour eux-mêmes. Dans le Devoll, seuls les Macédoniens de Vërnik semblent l'utiliser pour se désigner en Albanais. Dans une région majoritairement chrétienne orthodoxe comme la Lunxhëri, près de Gijrokastër, le mot kaur est au contraire beaucoup plus souvent entendu de la part des chrétiens, pour se distinguer des musulmans.
} 


\section{L'occupation de l'espace}

Le deuxième critère par lequel on peut reconnaître l'existence de communautés religieuses est celui de l'occupation de l'espace. La majorité des villages sont homogènes quant à la confession de leurs habitants. Il existe ainsi des villages musulmans et des villages chrétiens et, dans les quelques villages mixtes, musulmans et chrétiens vivent dans des quartiers (mëhallë ou lagje) distincts. Par ailleurs, la majorité des villages chrétiens sont des villages de montagne, alors que les villages musulmans sont pour la plupart situés en plaine. L'association des chrétiens à la montagne et des musulmans à la plaine est telle qu'il existe toujours dans les discours locaux des justifications aux quelques exceptions (c'est-à-dire les villages de plaine chrétiens et les villages de montagne musulmans), et que certains villages de montagne musulmans sont pris pour des villages chrétiens par les musulmans de la plaine qui n'y ont jamais mis les pieds et qui sont persuadés que toute la montagne est chrétienne.

[27] L'identification des chrétiens à la montagne a cependant ses propres limites, dans la mesure où eux-mêmes se disent originaires de la plaine, d'où ils racontent avoir été chassés par les musulmans, et où les musulmans de la plaine ne désignent jamais les chrétiens par le terme malok, qui signifie "montagnard", avec une nuance péjorative (le montagnard est un rustre et un imbécile. Le mot est formé sur mal, la montagne, avec le suffixe péjoratif -ok). Ce terme est au contraire réservé à ceux que l'on peut appeler, dans les conceptions locales, les authentiques habitants de la montagne, c'est-à-dire les bergers (qu'ils soient Valaques ou Albanais) et les Guègues du nord du pays. D'autre part, si la montagne en tant que telle est un espace d'arriération opposé à la civilisation de la plaine (ce qui apparait très bien dans la façon dont le sud de l'Albanie, en tant que pays de plaines, se perçoit face au nord montagneux), la montagne chrétienne est au contraire valorisée, tant par les chrétiens que par les musulmans, comme un espace de culture (parfois presque au même titre que la ville) opposé à la plaine rurale et arriérée. L'opposition entre chrétiens et musulmans semble donc ici se superposer à une opposition préexistante entre la montagne et la plaine et la modifier dans un sens nouveau.

Il faut signaler enfin que si l'association des musulmans à la plaine et des chrétiens à la montagne n'est pas spécifique au Devoll, ni même à l'Albanie ${ }^{6}$, la configuration géographique du Devoll d'une part (il s'agit d'un bassin entouré de montagnes) et le type d'habitat de l'autre (il s'agit exclusivement d'un habitat groupé et permanent ${ }^{\top}$ ) rendent la ségrégation spatiale entre chrétiens et musulmans particulièrement sensible, facilitant le sentiment de communauté entre

\footnotetext{
6 Voir Xavier BOUGAREL, 1998. La “ revanche des campagnes" : entre réalité sociologique et mythe nationaliste. Balkanologie 2 (1), pp. 17-35 ; voir et Marko ZIVKOVIC, 1997. Violent Highlanders and Peaceful Lowlanders. Uses and Abuses of Ethno-Geography in the Balkans from Versailles to Dayton. Replike, special issue, pp. 107-119.

${ }^{7}$ Les villages du Devoll sont des fshat, des villages agricoles, opposés aux katund des régions pastorales.
} 
les gens qui partagent le même habitat : les identifications religieuses se font moins au niveau individuel ou à celui des communautés religieuses entières qu'à celui des villages et de l'occupation de l'espace. De la même manière, les problèmes de coexistence entre chrétiens et musulmans sont surtout des problèmes de relations entre villages voisins (à propos des forêts, de l'irrigation, des récoltes), et un [28] village chrétien de montagne peut s'opposer à un autre village chrétien, situé dans la plaine, comme s'il s'agissait d'un village musulman : on accuse par exemple les habitants du village de plaine d'être sales, ce qui est une accusation régulièrement portée contre les musulmans, et on refuse d'y prendre des conjoints.

\section{La pratique matrimoniale}

Les relations entre chrétiens et musulmans se définissent également par une endogamie assez stricte de communauté religieuse. L'endogamie des communautés religieuses est suffisamment perçue comme un facteur de séparation entre les chrétiens et les musulmans pour avoir fait l'objet de l'attention du pouvoir communiste dans la lutte contre ce qu'il appelait les "survivances rétrogrades". Les mariages mixtes ont alors été fortement encouragés par le pouvoir et leur progression est présentée dans les textes officiels de l'époque comme le signe de la disparition de la religion dans la société albanaise : si chrétiens et musulmans se marient entre eux, peut-on dire, c'est qu'ils ont cessé d'être chrétiens et musulmans. C'est pourquoi aussi les couples mixtes appartiennent en général à des catégories de la population plus sensibles à ce type de discours et aux avantages matériels qui sont liés à l'obéissance aux recommandations du pouvoir (enseignants, petits fonctionnaires, intellectuels d'origine rurale et modeste), et moins au personnel paysan des coopératives, sur lequel l'idéologie n'avait pas les mêmes prises, et dont les chances de promotion étaient de toute façon très faibles. En ce sens, le mariage mixte a par ailleurs, aujourd'hui encore, une connotation militante et progressiste.

L'endogamie dont il est question ici consiste plus en un refus de donner des épouses à l'autre communauté plutôt qu'à un refus d'y prendre des épouses. En ce sens le problème n'est pas tant celui de l'appartenance confessionnelle des enfants issus du mariage mixte, qui, compte tenu du caractère patrilinéaire du système de parenté albanais, héritent toujours de celle de leur père et n'ont donc pas de problème de choix ou de double appartenance. Les enfants de couples mixtes sont en effet toujours solidaires de la communauté religieuse de leur père, ne serait-ce que par le fait de vivre dans un village de cette communauté (puisque la résidence est patrilocale) : c'est aussi sur la familiarité avec les façons d'être et de faire que se construit l'identité religieuse ${ }^{8}$.

[29] Puisque l'endogamie est ici un refus de donner des épouses, il Convient de distinguer l'identité des donneurs dans les cas de mariages mixtes. On remarque alors que les

${ }^{8}$ Les choses sont un peu différentes en milieu urbain, qui ne nous concerne pas ici. 
chrétiens mettent encore plus de résistance à donner que les musulmans, alors qu'ils acceptent de prendre : dans la plupart des couples mixtes, l'homme est chrétien et la femme est musulmane. L'inverse se rencontre beaucoup plus rarement et les chrétiennes mariées à des musulmans sont en général des catholiques originaires du nord de l'Albanie, donc étrangères à l'opposition locale entre chrétiens et musulmans. Dans cette situation, les preneurs de femmes sont valorisés : les chrétiens mariés à des musulmanes se vantent d'avoir pris leurs filles aux musulmans, alors que les musulmans se plaignent de ce que les chrétiens leur prennent leurs filles, mais refusent de leur donner leurs filles à eux, comme si elles étaient trop bien pour les musulmans. On peut noter aussi que la situation actuelle est l'inverse de celle qui prévalait avant l'indépendance, c'est-à-dire au temps de la prééminence institutionnelle des musulmans dans le cadre de la société ottomane : les musulmans prenaient alors des épouses chrétiennes, mais ne donnaient jamais leurs filles à des chrétiens (c'est ce que l'on remarque dans les généalogies musulmanes). Le statut relatif des musulmans et des chrétiens s'est renversé dans la société locale - les chrétiens sont désormais reconnus comme formant une élite - et les comportements matrimoniaux ont accompagné cette évolution.

Concernant toujours l'endogamie, il faut ajouter enfin qu'une pratique endogame ne constitue pas un problème pour les musulmans, largement majoritaires à l'échelle du district (la plupart des alliances sont nouées dans le district du Devoll et dans celui de Korçë), alors que les chrétiens, beaucoup moins nombreux, sont plus rapidement limités par l'exogamie de lignage qui leur interdit d'épouser leurs cousins jusqu'à des degrés éloignés, tant du côté du père que du côté de la mère. C'est pourquoi de nombreuses chrétiennes se marient en ville (en particulier à Korçë, qui est perçue comme une ville " chrétienne"), faute de trouver un conjoint permis au village, et que les jeunes qui partent faire des études en ville ont tendance à $\mathrm{y}$ rester et à ne pas revenir au village pour y prendre un conjoint. Cette caractéristique renforce encore les liens que la communauté chrétienne entretient avec le monde des villes et contribue à lui apporter l'image de "culture" et de "progrès" qui s'attache, ici comme ailleurs, aux grandes villes (les villes en question ici étant aussi bien les grandes villes albanaises, en particulier Korçë et Tiranë, que la ville de Thessalonique en [30] Grèce et les villes de vieille émigration albanaise aux États-Unis : Chicago, New York, Boston).

\section{Le rapport au pouvoir et à l'État}

Le quatrième critère de différenciation entre chrétiens et musulmans est celui des relations avec le pouvoir et l'État. Jusqu'à la création de l'État albanais, les musulmans bénéficiaient d'une situation privilégiée dans ce domaine, dans le cadre de l'Empire ottoman, organisé justement en fonction de l'opposition entre musulmans et non musulmans. Aujourd'hui encore, les chrétiens albanais parlent de cette époque comme d'une époque d'oppression, dans laquelle les musulmans avaient tous les droits et notamment, dans une 
région d'agriculture, celui de s'approprier la terre. La présence des chrétiens dans la montagne est liée à cette époque.

Avec la création de l'État albanais, l'opposition entre chrétiens et musulmans n'est plus pertinente au niveau des institutions ; elle est remplacée par d'autres oppositions, notamment, après la Seconde Guerre mondiale, par celle entre communistes et non communistes (ces derniers étant maintenus dans une position inférieure qui rappelle parfois celle des non musulmans dans le système ottoman). Depuis l'apparition du pluralisme démocratique au début des années 1990, de telles distinctions institutionnelles ont disparu, mais elles survivent dans la polarisation de la vie politique en deux camps opposés, celui des socialistes, dont le parti est l'ancien Parti du Travail (communiste), et celui des démocrates, dont le parti est le premier parti créé à la fin de la dictature communiste (décembre 1990). Est-il possible de retrouver dans les oppositions actuelles la trace de l'opposition entre musulmans et non musulmans qui caractérisait la société ottomane ? Peut-on dire par exemple que les chrétiens sont plutôt communistes et les musulmans plutôt démocrates ?

Sur le seul plan électoral, il est impossible de répondre à la question, par manque de statistiques sur l'appartenance religieuse (les dernières données fiables sont celles du recensement italien de 1942 qui donne, pour toute l'Albanie et avec peu de changement par rapport aux chiffres des années 1920 : musulmans 68,9\% ; orthodoxes 20,7\% ; catholiques 10,4\%. Les statistiques de 1991 donnent pour leur part 21\% de musulmans, $74 \%$ d'athées et $5 \%$ de chrétiens, mais, elles sont sur ce point contestables, notamment par la présence d'une catégorie "athée"). Au regard des résultats électoraux par districts, on peut néanmoins relever que, depuis 1991, date des premières élections libres, les régions de population [31] mixte du sud du pays semblent plus facilement voter en faveur des socialistes ${ }^{9}$. Cela ne suffit cependant pas à établir un lien entre communauté chrétienne et socialisme.

Sur le plan des discours et comportements locaux, on peut dire en revanche que l'opposition entre musulmans et chrétiens est parfois politique, même si les choses ne sont jamais posées une fois pour toutes. Il est indéniable par exemple que la société communiste a permis aux chrétiens du Devoll d'échapper à la domination numérique et statutaire — ou ce qu'il en restait - des musulmans : en niant l'existence des communautés religieuses, le système communiste supprimait en même temps la discrimination religieuse ; par le développement de l'enseignement et des activités administratives, il a également fourni aux chrétiens le moyen de quitter leurs villages pauvres de la montagne autrement que par l'émigration, désormais interdite (jusqu'à la guerre, les chrétiens étaient beaucoup plus nombreux à partir que les musulmans). Aujourd'hui encore, il est courant d'entendre dire dans le Devoll que les chrétiens font plus d'études que les musulmans, et, en comparaison avec leur part dans la population, les chrétiens sont surreprésentés dans les professions liées à l'enseignement (il est courant d'avoir

${ }_{9}^{9}$ Christian PIHET, 1998. Élections et partis en Albanie : une lecture géographique. Hérodote, revue de géographie et de géopolitique 90 , pp. 46-61. 
un directeur d'école et des instituteurs chrétiens dans un village musulman, l'inverse n'est pas vrai) de même que dans les postes à responsabilité de l'administration. Le village de Bradvicë est à cet égard exemplaire: village chrétien de montagne, vivant d'élevage et d'artisanat (en particulier de poterie), il s'est presque entièrement vidé de sa population au temps du communisme, lorsqu'un de ses habitants est devenu membre des plus hautes instances du Parti et, fort de sa situation, a fait monter tous ses parents et amis dans la capitale. On peut donc dire, dans une certaine mesure, que les chrétiens ont bénéficié des transformations de la société sur un modèle communiste relativement plus que les musulmans.

Il est pourtant impossible d'identifier les chrétiens aux communistes ; eux-mêmes s'en défendraient, et surtout, dans les représentations locales, l'image des musulmans et celle des communistes ont beaucoup trop de traits communs. Du point de vue chrétien par exemple, les musulmans et les communistes représentent une même menace contre la religion chrétienne : les fermetures d'églises de 1967, souvent accompagnées de destructions, sont mises sur le même plan que les conversions forcées et les destructions opérées par les musulmans à l'époque de l'Empire ottoman. De la même manière, la collectivisation a été vécue par les villages chrétiens comme une dépossession des [32] chrétiens en faveur des musulmans (dans le cadre des coopératives, les anciennes limites villageoises avaient disparues ; musulmans et chrétiens des villages voisins travaillaient indistinctement les mêmes terres), pratique qui renvoie aussi dans le discours chrétien à l'époque ottomane et à l'oppression de chrétiens par les musulmans pour l'accès à la terre. Enfin, les communistes d'hier sont vus aujourd'hui, pour beaucoup d'entre eux, comme des arrivistes incompétents, embrassant l'idéologie officielle pour des avantages matériels, tout comme les musulmans représentent aux yeux des chrétiens ceux qui se sont convertis à l'islam pour profiter des privilèges que leur accordaient les Turcs.

Ainsi, plus qu'une réelle politisation des communautés religieuses, on voit ici une politisation des représentations de chaque communauté religieuse, comme si l'identité de ces communautés se construisait aussi à partir d'oppositions tirées du domaine politique, mais sans qu'il y ait jamais adéquation, même approximative, entre une communauté et un segment de la société politique ou un parti politique. En d'autres termes, on peut dire que les chrétiens se posent comme démocrates lorsque leurs voisins musulmans sont communistes, ou perçus comme en majorité communistes (c'était le cas par exemple entre le village chrétien de Voskopojë et son voisin musulman de Krushovë lors des élections parlementaires de mai 1996), et ils se posent au contraire comme socialistes lorsque leurs voisins musulmans sont perçus comme en majorité démocrates ou ballistes (extrême droite), comme c'est le cas par exemple entre le village chrétien de Qytezë et le village musulman de Miras. On voit donc par là que des oppositions de type politiques sont utilisées pour construire les identités religieuses, mais que les communautés religieuses ne se posent pas elles-mêmes comme des communautés politiques. 


\section{Tradition et progrès}

Les thèmes du progrès et de l'évolution sont très présents dans la société albanaise : il faut y voir sans doute une conséquence des transformations rapides qu'a connues le pays depuis un siècle - les mêmes idées ont fait leur apparition dans la société ottomane au dixneuvième siècle - avec également l'influence des conceptions marxistes de l'histoire et de la société, largement répandues par la dictature communiste. Il existe ainsi une forte préoccupation, sinon une obsession, à propos des questions de progrès et d'évolution, et les différences, y compris celles entre chrétiens et musulmans, sont pensées en termes d'avance et de retard sur une ligne d'évolution. Dans cette perspective, les chrétiens ont l'avantage sur les [33] musulmans : par leurs liens avec les villes et avec l'étranger, en particulier avec l'Europe et l'Amérique, ils sont considérés, y compris par les musulmans, comme étant "en avance" et comme représentant le modèle à suivre pour rattraper le retard que l'Albanie a accumulé par rapport au reste du monde. Les musulmans de la plaine, parlant des villages chrétiens de la montagne, ne manquent pas de raconter que, dès les années1930, et grâce à l'argent ramené par l'émigration, ces villages chrétiens étaient déjà équipés d'éclairage public, de rues pavées et de l'eau courante, alors que les villages musulmans attendent toujours aujourd'hui de telles innovations. Les vêtements européens, le mobilier, les façons de manger ou même de danser qui sont aujourd'hui les plus répandus dans la région sont conçus comme des innovations empruntées par les musulmans à leurs voisins chrétiens. En contrepartie, ces derniers ne manquent pas d'insister sur le retard et l'arriération des musulmans, qui vivent et pensent encore comme "au temps de la Turquie" (në kobën e Turqisè), c'est-à-dire comme il y a longtemps.

La valorisation du progrès n'est cependant pas la seule attitude face au temps et au changement. Il faut considérer aussi ce qui se dit de la tradition, c'est-à-dire d'une certaine forme de résistance au changement. On se rend compte alors que musulmans et chrétiens s'opposent encore sur leur rapport à la tradition. Le progrès que l'on attribue aux chrétiens ne signifie pas ici un oubli des origines ou un rejet du passé, au contraire : de l'avis même des musulmans, les chrétiens ont su conserver intactes leurs traditions religieuses et possèdent également une bonne connaissance de leur histoire ; eux-mêmes au contraire reconnaissent avoir abandonné la plupart de leurs traditions religieuses et n'avoir pas su garder de traces de leur passé. Lors du recueil de généalogies chez les musulmans, ceux-ci se sont souvent excusés de ne pouvoir remonter bien loin dans leur histoire familiale, faute de documents, alors que les chrétiens, disent-ils, grâce aux registres tenus par l'église, seraient bien plus capables de dire d'où ils viennent. Dans d'autres cas, les généalogies s'arrêtent car l'informateur reconnaît qu'on ne lui a jamais parlé de tel ancêtre en ligne directe, qui était sans doute chrétien et qui, après sa conversion à l'islam, avait cherché à faire oublier son origine (de nombreuses conversions à l'islam n'ont eu lieu qu'au début du dix-neuvième siècle). Il en va de même des traditions religieuses, en particulier des fêtes annuelles qui en sont la manifestation la plus évidente pour l'autre communauté, et les musulmans reconnaissent qu'ils sont beaucoup moins attachés que 
les chrétiens [34] à leurs propre traditions religieuses : les fêtes et cérémonies musulmanes ne sont suivies que par les plus âgés; les fêtes chrétiennes rassemblent au contraire beaucoup plus de gens, dont de nombreux musulmans. Et les chrétiens ne manquent pas de critiquer les musulmans pour la désinvolture avec laquelle ils laissent se perdre leurs traditions religieuses.

Il existe donc deux manières de poser le rapport au temps, et dans les deux cas, les musulmans s'opposent aux chrétiens. La première, et la plus explicite, est celle de l'évolution et du progrès, dans laquelle les chrétiens sont ouvertement dits "en avance" (përpara) et les musulmans " en retard" (mbrapa). La seconde n'est pas directement perçue par les uns et les autres, mais elle apparait en particulier dans les discours sur la tradition : les chrétiens vivent dans le temps, ils sont maitres de leur temps, alors que les musulmans sont hors du temps. Les premiers savent d'où ils viennent et où ils vont, les seconds cherchent à l'oublier.

Il faut voir ici dans ce rapport au temps une caractéristique de la plupart des musulmans balkaniques, et non pas des seuls Albanais, dont l'origine est problématique : anciens chrétiens convertis, les musulmans restent entachés de cette "trahison originelle", sans pouvoir non plus se solidariser entièrement avec les autres populations musulmanes extra-balkaniques, qui font figure d'envahisseurs. À titre de comparaison avec les Musulmans de Bosnie, voici par exemple ce qu'écrit l'écrivain Mesa Selimovic ${ }^{10}$ : “Que sommes-nous alors?... Les hommes les plus compliqués qui soient au monde... Hier nous étions ce que nous souhaitons oublier aujourd'hui.... Nous avons été déracinés, mais non accueillis.... Confusément honteux de notre origine, de notre reniement aussi, nous refusons de regarder en arrière, nous n'avons pas devant nous où poser les yeux ; nous retenons alors le temps, redoutant toutes les solutions, quelles qu'elles soient."

En Albanie, ce rapport différent au temps est encore exprimé, plus prosaïquement, dans un reproche souvent fait par les chrétiens aux musulmans : ces derniers passeraient en effet la plupart de leur temps au café à ne rien faire, alors que les chrétiens savent organiser leur temps et faire par exemple la distinction entre le temps du travail et celui du loisir. La même chose est par ailleurs [35] dite de l'argent, que les musulmans dépensent sans compter mais que les chrétiens savent gérer et économiser.

\section{La nature de l'autre}

Les membres des deux communautés se voient attribuer certaines caractéristiques, naturelles et héréditaires, qui sont à rapprocher de l'usage de désigner ces communautés comme deux "races" distinctes. La première de ces caractéristiques, que l'on trouve exclusivement dans le discours des chrétiens, s'organise autour d'une opposition entre le

${ }^{10}$ Dans Le derviche et la mort. Paris, Gallimard, 1977. p. 286. Cité par Catherine Samary, 1995. Mouvante identité des Musulmans. Le Monde diplomatique, octobre 1995. p. 13. 
propre et le sale : les chrétiens sont propres (i pastër), les musulmans sont sales (i pisët). Cette opposition a d'abord une dimension physique, celle de l'odeur: pour les chrétiens, les musulmans ont une odeur, les maisons musulmanes ont une odeur; elle a ensuite une dimension morale : être propre, c'est aussi avoir une vie bien réglée et ordonnée, que la saleté vient menacer et corrompre.

Vient ensuite une deuxième opposition, que l'on retrouve avec des nuances différentes chez les uns et les autres. Du point de vue chrétien, elle prend la forme d'une opposition entre intelligence et bêtise : les chrétiens sont intelligents ( $i$ zgjuar, $i$ mençur) et les musulmans sont des imbéciles (budalla); du point de vue musulman, elle prend au contraire la forme d'une opposition entre l'honnêteté et la malice : les chrétiens sont peut-être plus intelligents, mais ils sont surtout fourbes et perfides ( $i$ pabesè), alors que les musulmans sont honnêtes et dignes de confiance; ce sont des gens de parole (e mbajnë fjalën). Encore une fois, ces représentations réciproques ne sont pas spécifiques aux musulmans et chrétiens albanais; on les retrouve par exemple entre les Grecs et les Turcs en Anatolie ${ }^{11}$.

Il n'est pas impossible de voir dans ces deux systèmes d'oppositions la conséquence de la différentiation socioprofessionnelle des deux communautés, notamment à partir du dixneuvième siècle : la saleté dont il est question ici est celle liée à une existence rurale et agricole. Qu'il s'agisse de l'odeur de ceux qui rentrent des champs, des tas de fumier laissés par les éleveurs, des rues de village boueuses et défoncées par le passage des troupeaux, tout ce qui entre dans la catégorie du sale relève en fait de l'agriculture, c'est-à-dire, dans le Devoll, du mode d'existence des musulmans. Car, en partie par manque de terre dans la [36] montagne, les chrétiens se sont tournés en nombre vers l'artisanat, le commerce et les professions intellectuelles ou libérales, se détachant ainsi du monde rural, souvent contre leur gré et en étant obligés de quitter leur village pour la ville. De la même manière, la perfidie, la fourberie et le mensonge sont des défauts souvent attribués aux commerçants, mais aussi à ceux qui détiennent le savoir ou le pouvoir (professions intellectuelles, fonctionnaires), ce que sont devenus, de plus en plus, les chrétiens par rapport aux musulmans. L'honnêteté et la droiture sont au contraire associées à la campagne.

Un autre système d'oppositions attribue dans les représentations des musulmans le courage (trimëri) aux musulmans et la lâcheté aux chrétiens, alors que dans les représentations chrétiennes le courage des musulmans devient violence et la lâcheté des chrétiens devient sangfroid et contrôle de soi. Pareillement, la générosité (bujari) que les musulmans s'attribuent devient du point de vue des chrétiens une attitude dépensière, entre gaspillage et étalage de richesse, alors que l'avarice qu'ils dénoncent chez les chrétiens est vue chez ces derniers comme de la bonne gestion (nikoqirllëe). Ainsi chaque comportement et son opposé prennent une valeur positive ou négative selon les communautés (tableaux 1 et 2).

11 On en trouve de nombreux exemples dans Elia KAZAN, Au-delà de la mer Égée, Paris, 1995. 


\section{Conclusion}

Les différents critères sur lesquels repose le sentiment de différence entre chrétiens et musulmans ont donc peu de choses à voir avec la religion en termes de croyances ou de pratiques. Sur ce point, les membres de chaque communauté se contentent de constater que les uns vont (ou allaient) à la mosquée (xhami), alors que les autres vont (ou allaient) à l'église (kishè). Aucun point de dogme n'est soulevé, la religion de l'autre n'est pas attaquée en ellemême, elle est au contraire plutôt respectée en tant que religion, à travers ses lieux saints (vakef) et ses fêtes calendaires. La distinction entre ce que relève de l'islam et du christianisme n'est d'ailleurs pas toujours très nette, comme dans la description d'un lieu saint fait par une interlocutrice musulmane :

"Un lieu saint (vakef) est comme une mosquée (si xhami), mais en plus petit : il n'y a qu'une seule pièce. Mais à l'intérieur, il y a ce qu'il y a dans une mosquée : des icônes (ikonè), des bougies (qir), et des choses de ce genre. Il n'y a pas de prêtre (hoxbè), les gens y vont quand ils veulent, comme à l'église (si në kishè)."

Dans ce domaine, les seuls reproches entendus viennent des chrétiens, selon lesquels les musulmans albanais, anciens chrétiens, [37] non seulement auraient trahi le Christ en se convertissant à l'islam, mais ne respecteraient même pas leur nouvelle religion : on les accuse d'être pa fe, sans religion. C'est ainsi que l'on explique la saleté des musulmans : "leur religion aussi leur demande d'être propres, mais ils ne la respectent pas". Le seul jugement sur la religion provient également des chrétiens : comparé à l'islam, le christianisme orthodoxe est supérieur parce qu'il est plus ancien, mais aussi parce qu'il a plus de "culture" : les pratiques y sont plus policées (le fait de fêter les prénoms maintient de bonnes relations au sein du village, on résout les conflits lors de ces fêtes où les porteurs du prénom du saint du jour reçoivent la visite de tout le village), la prière moins humiliante (on ne s'abaisse pas, ne s'agenouille pas), et les pays chrétiens sont dits plus développés que les pays musulmans.

D'autre part, la plupart de ces oppositions sont orientées et derrière cette orientation se retrouve toujours le concept de "culture", comme expression de la modernité et comme comportement exemplaire. En ce sens, les relations entre chrétiens et musulmans prennent la forme d'une rivalité dans l'accomplissement d'une certaine forme de comportement social beaucoup plus que dans celui de l'identité nationale. Si chaque communauté revendique un niveau de culture élevé et cherche à rabaisser celui de l'autre communauté, il n'en reste pas moins que l'orientation générale est en faveur des chrétiens, auxquels les musulmans euxmêmes n'hésitent pas à reconnaitre un rôle de vecteur de la culture, notamment en raison de leur association à l'émigration et au milieu urbain. Cela est visible notamment dans la fréquentation des lieux saints (la plupart des fêtes pluriconfessionnelles sont des fêtes chrétiennes auxquelles les musulmans participent, rarement l'inverse) et dans les mariages 
mixtes, où les chrétiens acceptent de prendre dans l'autre communauté, mais refusent de donner. Il n'est pas impossible que l'orientation présente de ces relations soit assez récente, de même que le concept de culture qui les sous-tend: le recueil de généalogies fait apparâttre, jusqu'au milieu du XXe siècle, des mariages mixtes orientés différemment (des musulmans épousent des chrétiennes) et des conversions à l'islam se produisent jusque dans les premières années du XXe siècle, alors que la tendance aujourd'hui est plutôt à un retour vers le christianisme.

Pour terminer, le recours à la différence religieuse n'est pas le seul moyen d'exprimer l'appartenance et l'image de soi dans le Devoll. Chrétiens et musulmans sont conscients d'appartenir également au "Sud" et de s'opposer en presque tout aux gens du [38] "Nord". Il ne s'agit pas ici à proprement parler de la division "ethnique" des Albanais en Guègues au Nord et Tosques au Sud, mais bien d'une façon de se définir face à un autre vers lequel on a tendance à rejeter ses propres défauts : d'une manière générale, les gens du Devoll voient le Nord comme les chrétiens perçoivent les musulmans : violents, arriérés, sales et paresseux. Nord et Sud ne définissent d'ailleurs pas deux grandes régions de peuplement séparées par une limite physique (le fleuve Shkumbin est censé tracer une frontière entre Guègues et Tosques), mais plutôt deux espaces porteurs de caractéristiques opposées : le Nord est perçu comme un espace fermé, voire renfermé, et donc arriéré, sale et inquiétant, le Sud, comme un espace ouvert, dynamique, propre et rassurant. Le glissement depuis l'opposition religieuse vers l'opposition spatiale est fréquent dans le discours des gens du Devoll, comme dans l'histoire suivante, racontée par un instituteur d'un village musulman :

“La montagne (malësi, c'est-à-dire ici "le Nord") a des coutumes strictes. La femme par exemple y travaille beaucoup plus dur. Dans le Sud au contraire, du fait de l'émigration en Amérique et en Australie, la position de la femme est plus libre, même si elle n'est pas émancipée comme à l'Ouest [de l'Europe]. L'émigration a aussi apporté des changements de mentalité. Un homme de Ziçisht [le village voisin, chrétien] avait émigré et avait fait fortune en Amérique. Quand il est rentré, on lui a demandé comment il avait fait fortune. Il a seulement répondu qu'il essuyait toujours son assiette avec la dernière bouchée de pain. Cela veut dire qu'il ne faut pas demander plus que ce dont on a besoin, pour en laisser ensuite la moitié. Il faut économiser. Ici, au contraire, on a l'habitude de dire à celui qui ne veut pas boire de café dans la maison où il est entré " ce n'est pas un café qui nous appauvrit" (një kafe nuk na varféron). Mais pourquoi lui donner un café s'il n'en veut pas, et si c'est pour en manquer le lendemain ? ${ }^{12} "$

Cet exemple est intéressant parce qu'il montre bien comment fonctionne l'opposition entre Nord et Sud : le narrateur commence par opposer le Nord, avec ses coutumes sévères, au

\footnotetext{
12 Il faut insister ici sur le fait que tout visiteur se voit servir un café dans la maison dans laquelle il entre, et que ce geste est conçu comme la base même de l'hospitalité : envisager d'économiser un café dans ces conditions est véritablement une entorse aux règles de l'hospitalité.
} 
Sud, dans lequel il se place lui-même, influencé par l'émigration et donc avec plus de "culture". Dans un premier temps, le Sud est donc le lieu de l'innovation et [39] de la raison: la générosité et l'hospitalité n'y sont pas poussées jusqu'à l'absurde, on cherche plutôt à économiser, et il s'oppose au Nord plus conservateur et donc plus arriéré. Mais dans un deuxième temps, une autre opposition se substitue à l'opposition entre Nord et Sud, c'est celle entre chrétiens et musulmans : l'innovation en question, un "changement de mentalité", devient le fait d'un "homme de Ziçisht", village entièrement chrétien, et la générosité à outrance (obliger l'hôte à boire un café même s'il le refuse) est rapportée à un "ici " qui désigne le village musulman du narrateur. Autrement dit, ce que ce dernier reprochait d'abord au Nord, depuis sa position d'homme du Sud, il se l'attribue ensuite à lui-même, en tant que musulman. Mais le rapport aux valeurs reste le même : ce qui est bon en tant que pratique du Sud le reste en tant que pratique chrétienne, ce qui est condamnable en tant que pratique du Nord le devient en tant que pratique musulmane.

La description des communautés religieuses qui précède est rarement acceptée par les Albanais eux-mêmes, en particulier parmi les élites lettrées et urbaines, pour lesquels la différence religieuse doit au contraire être minimisée parce qu'elle constitue une menace pour l'unité nationale. J'ai cherché à montrer ici que la question des relations entre chrétiens et musulmans ne se posait pas en termes de respect ou d'atteinte à l'unité nationale, mais plutôt en terme de définition de la différence dans laquelle les communautés religieuses laissées par l'organisation sociale ottomane sont mises à contribution, comme l'est, de manière similaire, l'orientation spatiale du pays.

\begin{tabular}{|c|c|}
\hline CHRETIENS & MUSULMANS \\
\hline SANG-FROID & VIOLENCE \\
\hline INTELLIGENCE & BETISE \\
\hline ECONOMIE & GASPILLAGE \\
\hline
\end{tabular}

1. Chrétiens et musulmans selon les chrétiens

\begin{tabular}{|c|c|}
\hline MUSULMANS & CHRETIENS \\
\hline COURAGE & LÂCHETE \\
\hline HONNETETE & FOURBERIE \\
\hline GENEROSITE & AVARICE \\
\hline
\end{tabular}

2. Musulmans et chrétiens selon les musulmans 